\title{
Analyses of Factors Influencing Liquid Experiments in Microtubes
}

\author{
Zhan-hua Silber-Li ${ }^{1}$,Hai-hang Cui ${ }^{1}$, Zheng $\mathrm{Xu}^{2}$,Yu-ping Tan $^{3}$, Li-ding Wang ${ }^{2}$
}

I State Key Laboratory of Nonlinear Mechanics (LNM), Institute of Mechanics, Chinese Academy of Sciences, Beijing, 100080, China

${ }^{2}$ MEMS Research Center, Dalian university of technology, Dalian, 116024

${ }^{3}$ Shanghai Institute of Applied Mathematics and Mechanics, Shanghai University, Shanghai 200072 ,

\begin{abstract}
The experiments of microflow are very difficult to handle, owing to a few factors influencing the measurement accuracy. Based on our experiences, a detailed introduction about the experimental uncertainty and the relative factors is presented in this paper. From the analysis of the experimental uncertainty, the measurement of microtube diameters obviously dominates the experimental precision. Several factors, for example, the evaporation on gas/liquid interfaces, the deformation of microtube, the compressibility of liquid, the viscous heating and the wall slip are discussed. According to our present experimental results, the flow characteristics driven by the lower pressure in microtubes with the diameter larger than $20 \mu \mathrm{m}$ agree well with the prediction from classical Hagen-Poiseuille (H-P) theory, while the viscosity vs. pressure relationship should be considered under a high pressures in the microtubes with $3 \sim 10 \mu$ diameters, and the precision depiction of shape is taken into account in the cone-shape microneedle with the outlet diameter $1 \sim 15 \mu \mathrm{m}$.
\end{abstract}

Keywords: Microchannels, Liquids, Microfluidic, Uncertainty

\section{Introduction}

The microfluid mechanics mainly focus on the domain with the dimension from $\mathrm{mm}$ to $\mathrm{nm}^{[1,2]}$. Typically, the flow in microelectromechanical systems (MEMS) refers to the flow that has characteristic length less than $1 \mathrm{~mm}$ but more than 1 micron. Fluid flows in such small tubes differ from those in macro scale fields and have the following characteristics: (1) The surface-to-volume ratio has increased to $10^{6} \mathrm{~m}^{-1}$, which substantially affects on the thermal and mass transports related to surfaces; (2) With scale decreasing, space gradients become remarkable, the physical factors related to the gradients of the temperature and the velocity will be more important; (3) Obviously, interface effects of solid/liquid or liquid/gas dominate in small devices.

The development of microfluidic put forwards many new questions. For instance, in order to promote detecting efficiency in protein biochip, chaos is used to enhance the mixing in complex micro channels; in the micro device of gene transfer, it depends on the flow characteristics of micro nozzle to quantitatively 
control gene ${ }^{[3]}$; the stability of liquid drop is a key at a nano-jet formation. In order to solve these problems, both experiments and theoretical analyses are necessary.

One of the current experimental methods is to compare the measured flow rate in a microchannel with that predicted one from classical Hagen-Poiseuille (H-P) equation ${ }^{[4-6]}$. Ho and Tai (1998) ${ }^{[1]}$ and Gal-el-Hak (1999) ${ }^{[21}$ have introduced some experiments of gas in detail. But for liquids, the experiments of microflows are very difficult to handle, owing to several factors influencing the measurement accuracy. In this paper, based on our recent experiments in $\mathrm{LNM}^{[7-9]}$, these factors i.e. the evaporation on gas/liquid interface, the deformation of microtube, the compressibility of liquid, the viscous heating and the wall slip will be discussed and their estimations are given. The experimental uncertainty is also important for a successful experiment, therefore the method of analyses uncertainty is introduced. Finally, the results about simple liquids, deionized water, polar and non-polar liquids, in microtubes with the diameter $3 \sim 20 \mu \mathrm{m}$ and the cone-shape microneedle with the outlet diameter $1 \sim 15 \mu \mathrm{m}$ are presented briefly.

\section{Experimental setup and methods}

\subsection{Theory of laminar flow in a tube}

In the case of steady laminar flow in a straight tube, the Navier-Stokes (N-S) equation can be simplified as $\mathrm{H}-\mathrm{P}$ flow. The flow rate $Q_{H P}$ is expressed as: ${ }^{[10]}$ :

$$
Q_{H P}=\frac{\pi \mathrm{d}^{4}}{128 \mu L} P
$$

where $d$ and $L$ are the diameter and the length of the microtube, respectively, $P$ is the pressure drop between the two ends of the tube and $\mu_{i}$ the viscosity. The normalized friction coefficient $C^{*}$ is defined as:

$$
C=\frac{f_{\exp } \operatorname{Re}}{f_{H P} \operatorname{Re}}=\frac{Q_{H P}}{Q_{\exp }}
$$

where $f_{H P}$ and $f_{\text {exp }}$ are the theoretical and the experimental friction coefficient respectively. It is conventional to calculate $C^{*}$ with measured flow rate. If the relation of flow rate and pressure agrees with the prediction from H-P equation, the experimental flow $Q_{\exp }$ will equal to the theoretical flow $Q_{H P}$, it means $C^{*}=1$. If $Q_{\exp } \neq Q_{H P}$, then $C^{*}$ will deviate from 1 which is used to describe the difference between $Q_{\exp }$ and $Q_{H P}$.

\subsection{Experimental setup}

Our experiment was carried out in a microflow setup of LNM laboratory (Fig. 1)

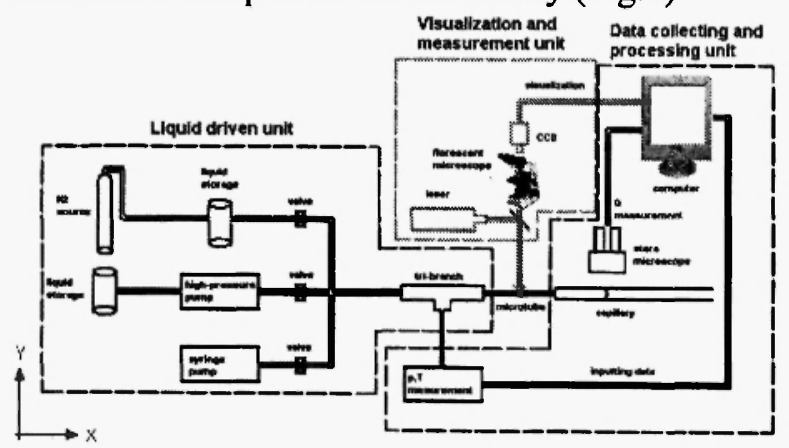

Fig. 1.A sketch of the high-pressure microflow setup

The experimental setup includes three parts: the pressure source, the visualization and measurement unit and the data collecting and processing unit. According to different driven pressures, the experimental setups are classified into the low-pressure one and the high-pressure one. For the former with pressure ranging from 0 
to $1 \mathrm{MPa}$, the nitrogen after modulating and filtering acts as the pressure source to drive the liquid in storage. The latter with pressure ranging from 1 to $40 \mathrm{MPa}$, a liquid pump is used to drive the working liquid directly. The pressure transducer and the thermocouple are connected to the tee connector, through which the inlet pressure of the microtube and the temperature of the working liquid can be measured. One end of the microtube is linked to the tee connector with a mechanical seal and the other end is inserted into a glass capillary with epoxy glues. The working liquid passing through the microtube is gathered in the glass capillary. Through measuring the reference interface displacement of the liquid column in the capillary $s$ and the interval $t$, the flow rate $Q_{m ;}$ can be calculated by the following equation:

$$
Q_{\exp }=\frac{\pi D^{2} s}{4 t}
$$

where $D$ is the diameter of the capillary.

\subsection{The uncertainty of experimental system}

From the above analysis, all the factors that may be affect $C^{*}$ are pressure drop $P$, viscosity $\mu$, the diameter and the length of microtube $d$ and $L$, the diameter of capillary $D$, the displacement of the interface in the capillary $s$ and the interval time $t$. Therefore, the total uncertainty of the experimental system depends on the measurment accuracy of all above parameters. The final expression of the uncertainty of $C^{*}$ is defined as:

$$
\frac{u_{\tau^{*}}}{C^{6}}=+\sqrt{\frac{16}{d^{2}} u_{i}^{2} \div \frac{u_{P}^{2}}{P^{2}}+\frac{u_{i}^{2}}{\mu_{0}^{2}}+\frac{u_{L}^{2}}{L^{2}}: \frac{4}{D^{2}} \cdots_{D}^{2} \div \frac{u_{s}^{2}}{s^{2}} \div \frac{u_{t}^{2}}{t^{2}}}
$$

where $u_{i}$ in the radical sign is the uncertainty of the related parameter referring to the respective subscripts. The uncertainties of the parameters, except the diameter of microtubes, are approximately constants in each experiment. The experimental uncertainties are listed in TABLE 1.

\begin{tabular}{|c|c|c|c|c|c|c|c|c|}
\hline Microtube & \multicolumn{7}{|c|}{ Relative uncertainties of parameters } & \multirow{2}{*}{$\begin{array}{l}\text { Uncertaint } \\
\text { y of } \\
\text { experimen } \\
\text { al system }\end{array}$} \\
\hline & $d$ & $P$ & $\mu$ & $\mathbf{L}$ & $D$ & $\boldsymbol{s}$ & $t$ & \\
\hline 10.02 & $\pm 1 \%$ & \multirow{3}{*}{$\begin{array}{c} \pm 0.3 \\
\%\end{array}$} & \multirow{3}{*}{$\begin{array}{c} \pm 0.75 \\
\%\end{array}$} & \multirow{3}{*}{ $\pm 0.2 \%$} & \multirow{3}{*}{ $\pm 0.5 \%$} & \multirow{3}{*}{ $\pm 1 \%$} & \multirow{3}{*}{$\begin{array}{c} \pm 0.2 \\
\%\end{array}$} & $\pm 4.5 \%$ \\
\hline 4.99 & $\pm 2 \%$ & & & & & & & $\pm 8.3 \%$ \\
\hline 2.95 & $\pm 3.3 \%$ & & & & & & & $\pm 13.4 \%$ \\
\hline
\end{tabular}

TABLE 1. Uncertainties of the experimental system

From equation (1), the flow rate is proportional to the fourth power of diameter. The uncertainty in the geometric measurement becomes very important from equation (4). The Scanning Electronic Microscopes (JSM-5600 LV and CSM950) with a precision of $0.1 \mu \mathrm{m}$ are used and several cross-sections of microtube are measured to verify the distribution of the diameter along the axis. For $10 \mu \mathrm{m}$ microtube, the total uncertainty is less than $\pm 4.5 \%$, in which the relative uncertainty of measuring diameter reaches up $4 \%$. But for smaller microtubes, this method should be improved.

\subsection{Some factors in microflow measurement}

Besides the system uncertainty, there are 
several factors that may influence the experimental data: the evaporation, the deformation of the microtube, the compressibility of the liquids, the wall slip and the viscosity etc. They will be discussed in detail as follows:

\subsubsection{Evaporation}

When a capillary is used to measure the flow rate, the vaporization in interface 2 would bring about the error (Fig.2). The quantity of liquid evaporation is related to the relative humidity $R H$ and environmental temperature $T$, and the area of capillary. The difference between $T$ and dew point temperature $T_{D}$ results in the heat exchange at the interface of liquid/gas, and the absorbing heat equals to the multiplication evaporation amount of liquid mass by latent heat of evaporation $Q$. The following equation approximately depicted this equilibrium:

$$
m \cdot Q \sim k_{\text {air }}\left(T-T_{D}\right) \cdot d
$$

$k_{\text {arr }}$ is the thermal conductivity of air $(0.028 \mathrm{w} / \mathrm{m} . \mathrm{k}), d$ is the characteristic dimension of microtube. As $d=10 \mathrm{um}, T=20 \mathrm{C}, R H=80 \%$, the evaporation amount of liquid mass equals to $0.05 \mathrm{nl} / \mathrm{s}$ approximately. Comparing with the current flow rates ranging from $0.1 \mathrm{nl} / \mathrm{s}$ to $100 \mathrm{nl} / \mathrm{s}$, the evaporation can be neglected. Although, an improvement method is proposed: the interface 1 of a gas bubble instead of interface 2 is used as the reference interface. This method ensures no additional evaporation during the measurement.

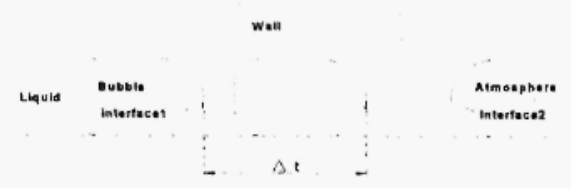

Fig.2. The principle of flow rate measurement.

\subsubsection{Roughness}

The roughness inside a microtube can be measured with Atomic Force Microscope. The relative roughness $R a$ is defined as:

$$
R a=\frac{\Delta h}{R} \times 100 \%
$$

where $\Delta h$ is the average height of the roughness peak and $R$ is the radius of the microtube. Two kinds of microtubes, $10 \mu \mathrm{m}$ and $500 \mu \mathrm{m}$, made by the same company with the same material are used. The average height $\Delta h$ ranges from $7.12 \mathrm{~nm}$ to $70 \mathrm{~nm}$, the relative surface roughness is calculated to be less than $0.7 \%$. So the microtube can be regarded as the hydraulic smooth tube.

\subsubsection{Effects of deformation \& compressibility}

The microtube is treated as a thick wall cylinder and its radial expansion $y(r)$ can be calculated as ${ }^{[11]}$,

$$
y(r)=\frac{d^{2} P}{E\left(d_{\text {outer }}^{2}-d^{2}\right)}\left[\frac{(1+v) d_{\text {ouler }}^{2}}{r}+(1-v) r\right]
$$

where $d_{\text {outer }}$ is the outer diameter of the microtube, $E$ the Young's modulus of the glass $\left(6.2 \times 10^{11} \mathrm{dyn} / \mathrm{cm}^{2}\right), \quad v$ Poisson ratio. In the case of $10 \mu \mathrm{m}$ microtube with the maximum pressure of $30 \mathrm{MPa}$, the radial expansion is $6 \mathrm{~nm}$ and the relative change of the diameter is only $0.06 \%$. Therefore, the influence of the deformation on the flow rate can be neglected.

It is well known that liquids can be compressed under a high pressure and Mach number may be served as a scale of the compressibility. According to the relation of Mach number and the density of the liquids, in the case of the maximum pressure of $30 \mathrm{MPa}$, the maximum velocity of the liquid in $10 \mu \mathrm{m}$ microtube is smaller than $2 \mathrm{~m} / \mathrm{s}$ and the Mach number is near $2 \times 10^{-3}$. Therefore we can obtain 
the relative change of the density, $\Delta \rho / \rho_{0}<0.001 \%$, which can be neglected too.

\subsubsection{Viscous heating}

In the case of a steady flow, the order of magnitude of the mechanical energy loss rate per unit volume due to viscous effects $\dot{\mu r}^{2}$ is equal to that of the internal energy change rate due to heat conduction $\kappa \Delta T / R^{2}$ according to the energy equation, ${ }^{15}$ where $\dot{\gamma}$ and $R$ are the typical values of the shear rate and the system size, respectively, $T$ is the temperature and $k$ is the thermal conductivity of the fluid. It is then straightforward to obtain the order of magnitude for the relative change of viscosity due to viscous heating:

$$
\frac{\mu}{\mu_{0}} \approx \frac{\beta}{T_{0}} \frac{\mu}{\kappa}(R \dot{\gamma})^{2} \sim \mathrm{O}\left(10^{-5}\right)
$$

where $\beta$ is the property coefficient, therefore the viscous heating can be neglected.

\subsubsection{Wall slip}

As the characteristic scale of the flow goes down to the micron/nanometer, the interaction between fluid and solid surfaces becomes more and more important. Based on the similar experimental condition, Choi etc ${ }^{[12]}$ proposed a formula to calculate the slip length $\delta$ and slip velocity $_{u_{\mathrm{v}, p}}$ as a function of shear rate $\dot{\gamma}$ :

$$
\delta=0.059 \times \gamma^{.04 \times 5}, u_{\frac{i, \pi}{,}}=\delta \cdot \gamma_{\text {wall }}=\delta \cdot \frac{P \cdot d}{4 \mu_{0} L}
$$

where the parameters are the same as noted in the formula (1). The relative change of flow rate is defined as:

$$
Q_{m \ldots d_{r}}=u_{\dot{H}_{r}} \frac{\pi d^{2}}{4}+\frac{\pi d^{v}}{128 \mu_{0} L} \Delta P
$$

$$
\frac{Q_{I t r-s i p}-Q_{H P}}{Q_{H P}}=\frac{8 \delta}{d}
$$

In case of the maximum pressure $30 \mathrm{MPa}$, the wall slip results in about $5 \%$ increase of the flow rate in $10 \mu \mathrm{m}$ microtube.

\subsubsection{Relationship of viscosity vs. pressure}

P.W.Bridgman carried out systematic experiments to study the relationship of viscosity vs. pressure. He pointed out that the viscosity of liquids, except the water, augmented with the increasing of pressure. The relationship can be expressed approximately by an exponential function: ${ }^{[13]}$ :

$$
\mu_{\text {app }}=\mu_{0} e^{x^{P}}
$$

where $\mu_{0}$ is the viscosity of the atmospheric temperature, $\mu_{\text {sp }}$ the apparent viscosity and $\alpha$ is the property coefficient. The ratio of the revised flow rate $Q_{H P \text { - } \mu}$ to the flow rate $Q_{H P}$, is,

$$
\frac{Q_{t r-\beta \mu}}{Q_{t r}} \approx\left(1-\frac{\alpha P}{2}\right)
$$

The viscosity for water in the present pressure range can be approximately regarded as a constant. For isopropanol and carbon tetrachloride, $\alpha$ are $7.90 \times 10^{-3} \mathrm{MPa}^{-1}$ and $8.08 \times 10^{-3} \mathrm{MPa}^{-1}$, respectively. When the maximum pressure $30 \mathrm{MPa}$ is applied, about $12 \%$ decreasing of flow rate is acquired.

\section{Experimental results}

As mentioned above, the system uncertainty of our experiments and the relative factors have been analyzed seriously, the experimental results obtained in LNM will be summarized as follow:

\subsection{Results of microtubes}

\subsubsection{Results of low pressure}

With the micro-flow experimental apparatus of pressure ranging from 0 to $1 \mathrm{MPa}$, the simple liquids with small molecules like deionized 
water and polar or non-polar organic liquids $\left(\mathrm{CCL}_{4}, \mathrm{C}_{6} \mathrm{H}_{5} \mathrm{C}_{2} \mathrm{H}_{5}\right.$ and isopropyl alcohol etc.) have been investigated. The results show that the flow rate behaviors in microtubes with diameters over than $20 \mu \mathrm{m}$ agree with the conventional theory of H-P flow ${ }^{[7,8]}$. These results conflict with the prior result of Pfahler ${ }^{[4]}$, but it is supported by the updated experimental results [14].

\subsubsection{Results of high pressure}

As the flow rate is proportional to the fourth power of the diameter, a pressure up to 1 30MPa must be maintained in order to accurately measure the flow rate in microtubes with 3-10um diameters. We still focus on the same simple liquids. The flow behavior of isopropanol and $\mathrm{CCL}_{4}$ in $3 \sim 10 \mu \mathrm{m}$ microtubes deviates from the classical H-P law, while water does not show the similar phenomenon. As the analyses above, it may be seen that the viscosity at high pressure plays an important role here. An exponential function of viscosity vs. pressure is introduced into $\mathrm{H}-\mathrm{P}$ equation to counteract the difference between experimental and theoretical values $^{[9]}$.

\subsection{Results of cone-shape microneedle}

The flow characteristics of a cone-shape microtube involve the injection precision of gene transfer in the micro device. It is difficult to control very tiny liquid $1 \sim 2 n \ell$ in each injection, the operator only relies on the deformation of a cell to judge the dosage indirectly. It is significant to design a kind of automatic apparatus to complete the injection process ${ }^{[3]}$. The microneedle is made with its inlet diameter $0.6 \mathrm{~mm}$ and outlet diameter ranging from $1 \sim$ $15 \mu \mathrm{m}$, the cone angle between 0 and $10^{\circ}$. Through introducing the variation of angles along the axe, the relationship of pressure vs. flow rate agrees much better than the fixed angle model $^{[15]}$.

\section{Conclusions}

In microfluidic experiments, the flow characteristic dimension concerns about micron. In the experimental research, how to realize the measuring accurate is extreme important and some details which often is neglected in macroscopic experiments have to be reconsidered:

1. The measurement precision of microtube diameters obviously dominates the experimental uncertainty;

2. Under our experimental condition, the evaporation, the viscous heating, the compressibility of liquids and the deformation of microchannel can be neglected, while the relationship of viscosity vs. pressure and the wall slip (maybe induced by roughness, wettingbility etc.) are taken into account;

3. According to the present results of simple liquids, it is remarkable that H-P law is suitable in microtubes with diameter larger than $20 \mu \mathrm{m}$. With the increasing of applied pressure, the relationship of viscosity vs. pressure and the wall slip should be considered and the relevant revisions are introduced into the H-P equation to counteract the difference of experimental results;

4. The function of angle variation along the axe is introduced into H-P equation to calculate the flow rate in core-shape micro needle, and a better agreement with experimental results is achieved.

Microflow as a recent research field involves micro heat transfer, micro/nano tribology and surface physics and chemistry etc. It is obviously a challenge to develop specious 
experimental methods and to reveal the non-linear phenomena in future research.

\section{Acknowledgements}

Thanks are also due to National Foundation Research Item (G1999033106) and Chinese Academy of Sciences Major Innovation Project (KJCX2-SW-L2) and National Natural Science Foundation of China (10272107) for their support to this research.

\section{Reference}

1. Ho Chih-Ming, Tai Yu-Chong. Micro-electro-mechanical-systems (MEMS) and fluid flows, Annu. Rev. Fluid Mech., 30(1998), 579-612;

2. Gad-el-Hak M. Handbook of MEMS, CRC Press, Boca Raton, 2001

3. Wang Li-ting, Liu Chong. Study about micro stereoscopic images of micro operation system (in Chinese), $J$. of Instruments, 20(1999), 165-167

4. Pfahler J., Harley J., Bau H. and Zemel J. Gas and Liquid Flow in Small Channels, DSC, 32(1991), 49-59

5. Jiang X.N., Zhou Z.Y., Yao J., Li Y., and Ye X.Y. Micro-Fluid Flow in Microchannel, Transducers'95, 1995, EurosensorsIX, Sweden, 317-320

6. Mala G.M. Dongqing Li. low Characteristics of water in microtubes, Int. J.of Heat and Fluid Flow, 20(1999), 142-148;
7. Li Zhanhua, Zhou Xinbei and Zhu Shannong. Flow characteristics of non-polar organic with small molecules in a microchannel, ACTA Mechanica Sin. (in Chinese), 3(2002), 432.

8. Li Zhanhua and Cui Haihang. Proceeding of experiments about liquid flow through micro-tubes, Inter J. Nonlinear Sci. And Num. Simulation, 3 4(2001), 577

9. Cui Haihang, Li Zhanhua and Zhu Shannong. Flow characteristics of liquids in microtubes driven by a high pressure. Physics of Fluids, Vol.16, No5, May 2004

10. Landau L.D. and Lifshitz E.M. Fluid Mechanics, Second edition, Butterworth Heinemann Press, 1987, 530

11. Landau L.D. and Lifshitz E.M., Theory of Elasticity, Pergamon Press, 1986

12. Choi Chang-Hwan, Johan K., Westin A. and Breuer K.S. Apparent slip flows in hydrophilic and hydrophobic microchannels, Physics of Fluids, 15(2003), 2897-2902

13. P.W.Bridgman, in physics of high pressure, G.Bell and Sons Ltd, 1952

14. Sharp, K.V., Adrian, R. J., Santiago, J.G. and J.I Molho. Liquid flows in microchannels, in Handbook of MEMS, CRC Press, Boca Raton, 2001

15. Xu Zhen, Li Zhan-hua, Liu Chong, Cui Hai-hang and Wang Li-ding. Experimental research on flow characteristics in microinjection (in Chinese), Accepted by $J$ of Applied Mechanics 